\title{
Feasibility study of new natural leaf-derived surfactants on the IFT in an oil-aqueous system: experimental investigation
}

\author{
Ali Khorram Ghahfarokhi • Abbas Dadashi • \\ Amin Daryasafar $\cdot$ Jamshid Moghadasi
}

Received: 3 August 2014/Accepted: 2 February 2015/Published online: 14 February 2015

(C) The Author(s) 2015. This article is published with open access at Springerlink.com

\begin{abstract}
Surfactant flooding is an Enhanced Oil Recovery technique used for decreasing oil trapping in pore spaces by reducing the interfacial tension (IFT) between oil and water. In this study, three types of plants based on natural cationic surfactants, named Olive, Spistan and Prosopis, are introduced and the application of these natural surfactants in reducing the interfacial tension of wateroil system is investigated. For this purpose, three naturalbased surfactants were extracted from the leaves of the trees of addressed plants and then the interfacial tension values between oil and natural surfactant solution were measured using the pendant drop method. The results demonstrated that Olive extract was able to lower the IFT between kerosene and distilled water from 36.5 to $14 \mathrm{mN} /$ $\mathrm{m}$, while Spistan and Prosopis extract could reduce the IFT from 36.5 to $20.15 \mathrm{mN} / \mathrm{m}$ and 36.5 to $15.11 \mathrm{mN} / \mathrm{m}$, respectively. According to these results in addition to the low price of generating natural surfactants, the feasibility of using these kinds of surfactants in future oil recovery processes is of major concern.
\end{abstract}

Keywords Surfactant flooding - Interfacial tension · Natural surfactants · Olive leaf · Spistan leaf · Prosopis leaf

\section{Introduction}

Surfactant flooding is an Enhanced Oil Recovery (EOR) technique which its objective is to decrease oil trapping by

A. Khorram Ghahfarokhi $(\bowtie) \cdot$ A. Dadashi · A. Daryasafar . J. Moghadasi

Department of Petroleum Engineering, Ahwaz Faculty of Petroleum Engineering, Petroleum University of Technology, Ahwaz, Iran

e-mail: alikhorram69@yahoo.com reducing the interfacial tension of water and oil system and so can improve the water flooding sweep efficiency.

Surfactant flooding can be affected by different parameters such as surfactant cost, interfacial tension reduction, oil recovery and etc., to be an economical process (Spildo et al. 2012). Nowadays because of environmental fates, natural-based surfactants can be used instead of industrial surfactants and many researchers have studied on these kinds of surfactants in recent years. The first natural surfactant was Quillaja Saponaria Molina that was extracted from a Chilean Soar bark tree (Rigano and Lionetti 2009). Shahri et al. (2012) used Amott cell to extract a natural surfactant from Zyziphus Spina Christi and introduced the application of this surfactant in oil recovery. After that, Ahmadi and Shadizadeh $(2012,2013)$ investigated the adsorption behavior of this surfactant onto carbonate rocks at different conditions. Deymeh et al. (2012) introduced a new natural cationic surfactant, named Seidlitzia rosmarinus and investigated the viability of using this surfactant for reducing Interfacial Tension (IFT) in surfactant flooding processes. Seidlitzia rosmarinus surfactant with Critical Micelle Concentration (CMC) value of about $8 \%$ by weight imposes a considerable decrease in oil-water interfacial tension. Ahmadi et al. (2014) formulated a micro-fluid using micro-sized particles of mulberry leaf and studied the effect of mulberry leaf-derived surfactant on the IFT between oil and water at different surfactant concentrations. They demonstrated that a microfluid containing $1 \mathrm{wt} \%$ of mulberry leaf particles could reduce the IFT between distilled water and kerosene by $60 \%$.

In this paper, three new natural cationic surfactants produced from the leaves of Olive, Spistan and Prosopis, are introduced. Massive efforts have been put forth to study the effect of these surfactants on the interfacial tension 
between water and oil. For this purpose, surfactants were extracted from the leaves of the trees of addressed plants and pendant drop method was then employed to measure the IFT between the surfactant solution and oil.

\section{Materials and experimental procedures}

Materials

\section{Aqueous phase}

In experiments, deionized water is used for measuring interfacial tension since natural surfactants will precipitate in brine.

\section{Surfactant}

Olive leaf-derived surfactant The olive tree is a short, evergreen tree, and rarely exceeds $26-49 \mathrm{ft}$ in height. It has leaves (Fig. 1) which are oblong and silvery green, about 1.6-3.9 in. long and 0.39-1.18 in. wide.

Alibes and Berge (1983) analyzed the compositions of air-dried olive leaves. The chemical compositions in the form of gross composition are as follows: $95 \%$ Dry matter, $95 \%$ Organic matter, 7-11\% Crude protein, 13-23\% Crude fiber, $5 \%$ Ether extract, 40-45\% Neutral detergent fiber, $28-35 \%$ Acid detergent fiber and $18 \%$ Acid detergent lignocellulose [8].

The olive tree is now been widely available in several regions such as northern Iran at the south of the Caspian Sea, eastern Mediterranean Basin, northern Saudi Arabia and northern Iraq. The olive tree is the sole source that provides the health benefits of olive leaf extract, also it has leaves that can be used as a source for generating surfactants.

Spistan leaf- derived surfactant Cordia myxa, also known as Spistan, is a species of plant which is a mediumsized and deciduous tree. The tree has a main trunk which

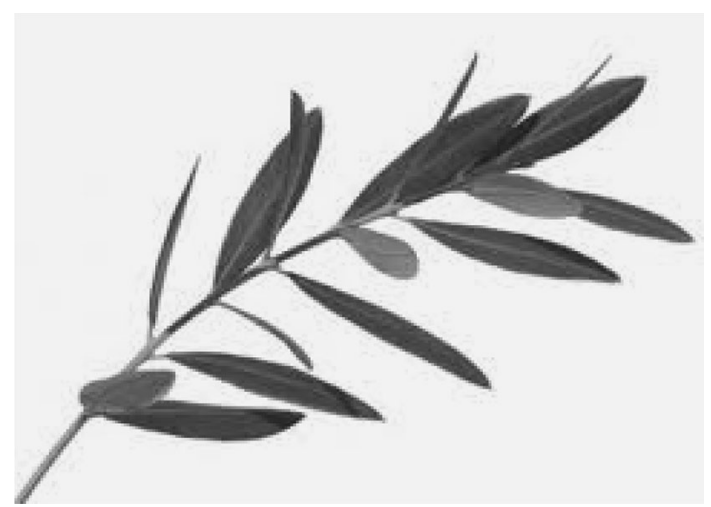

Fig. 1 Olive tree leaf is straight and cylindrical with a height of nearly $4 \mathrm{~m}$. The height of the fully grown-up tree becomes $10-15 \mathrm{~m}$. The leaves of Spistan (Fig. 2) are glabrous above and pubescent below with the spread being $10 \mathrm{~cm} \times 7 \mathrm{~cm}$.

The Spistan tree is the source that provides the health benefits such as protective role against liver fibrosis, and its leaves' extract can be used as a resource for generating antioxidants and surfactants.

Spistan fruits are found to be medium in crude protein (8.32\%), carbohydrate $(57.08 \%)$, ash $(6.7 \%)$, fiber $(25.7 \%)$ and fat $(2.2 \%)$ and its leaves contain crude protein, crude fiber, carbohydrate, lipid and ash (Aberoumand, 2011).

Prosopis leaf-derived surfactant Prosopis is a perennialand medium-sized tree which can grow to $30-50 \mathrm{ft}$ tall. It has 4-7 in. long and fine leaves (Fig. 3). Dry Prosopis leaves contain protein, carbohydrate, lipid, crude fiber, ash and moisture and have properties that can be used as surfactants.

\section{Oil}

In this study, kerosene was used as the oil phase since in interfacial tension measurements, the oil phase is better to be clean. The density of kerosene at a test temperature of $24.2{ }^{\circ} \mathrm{C}$ was $0.7769 \mathrm{~g} / \mathrm{cm}^{3}$.

Experimental procedure

\section{Extract preparation}

The leaves of three types of plants (olive, Spistan and Prosopis) were hand-picked from trees, dried at ambient

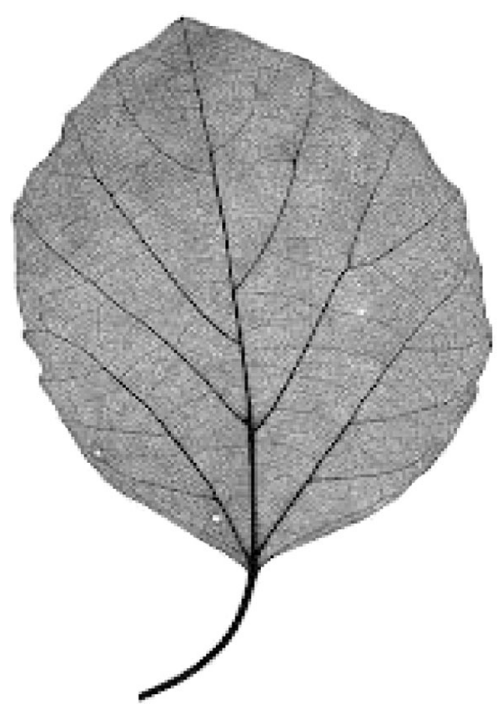

Fig. 2 Spistan tree leaf 


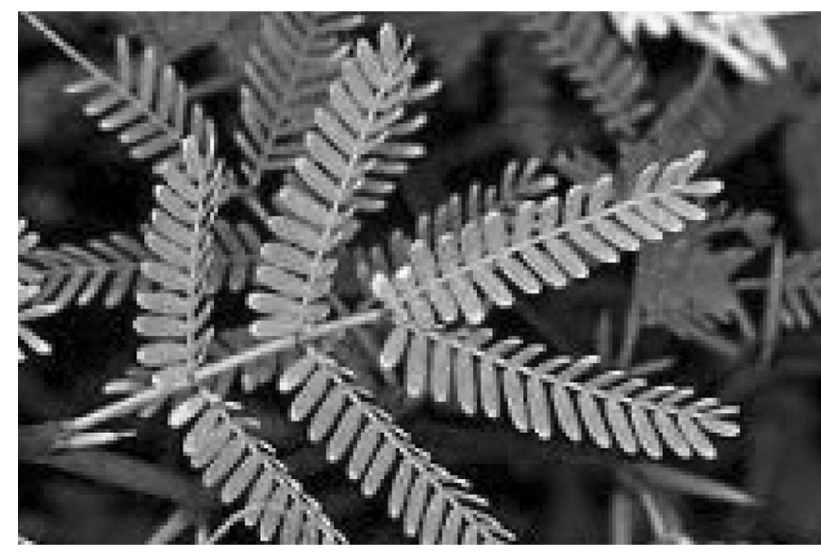

Fig. 3 Prosopis tree leaf

condition. Powder of dried leaves was inserted in Soxhlet extractor and after about $12 \mathrm{~h}$, a viscous solution was appeared in the chamber of the Soxhlet extractor. The viscous solution was then put in oven with temperature of $76^{\circ} \mathrm{C}$ so the little amount of remained water could be vaporized without damaging the extract. We used the obtained extract of each plant to make solutions with known concentration for investigating the effect of different quantities of the derived extract in the surfactant's ability in lowering interfacial tension.

\section{CMC measurement}

There are several methods to measure CMC; in this study conductivity method was used. For this purpose, conductivity of natural surfactant solutions was determined using a conductivity detector which is from Crison Company. At first the conductivity detector was calibrated using standard solution and the probe was washed up with distilled water for accuracy of solutions' conductance. The value of CMC can be figured out from the turning point in the plot of conductivity versus concentration. Surfactant ions or molecules will start to become micelles when surfactant solution concentration increases to a certain value, this causes a change in trend of curve. Figures 4, 5 and 6 are the plots of conductivity versus solution concentration for Olive, Spistan and Prosopis, respectively.

Olive-derived surfactant has a critical micelle concentration of about $1.95 \%$ by weight, while Spistan-derived surfactant and Prosopis-derived surfactant have CMCs of about 2.1 and $2.3 \%$, respectively.

\section{Method accomplishment in measuring interfacial tension}

Pendant drop method was used for IFT measurements using the Captive Drop Instrument. Pendant drop method needs the density values of surfactant solution and oil for measuring the IFT between oil and natural surfactant solution. The densities of different surfactant solutions were measured using Density Meter Apparatus at ambient pressure and temperature. The densities of different percent weights of aqueous solutions of olive, Spistan and Prosopis extracts are illustrated in Tables 1, 2 and 3, respectively. Kerosene was used as the oil phase. The pump of the pendant drop instrument was adjusted to inject surfactant solution so that the low flow rate ensures that releasing the oil drop is the result of density difference. This method utilizes a procedure which is based on Axisymmetric Drop Shape Analysis (ADSA) that uses the shape of drops to measure the interfacial tension. In this study, an accurate method described by Herd et al. (1992) was used to calculate the interfacial tension.

The interfacial tension is:

$\sigma=\frac{\Delta \rho g R_{0}^{2}}{\beta}$

where $\sigma$ is the interfacial tension, $\Delta \rho$ is the density difference between two phases and $\mathrm{g}$ is the acceleration due to gravity. $\beta$ and $R_{0}$ are determined as follows:

$\beta=0.12836-0.7577 S+1.7713 S^{2}-0.5426 S^{3}$

$\frac{d}{2 R_{0}}=0.9987-0.1971 \beta-0.0734 \beta^{2}+0.34798 \beta^{2}$

$S$ is the drop shape factor and equals to d/D. D is the maximum diameter of the drop and used in the calculation of the drop's horizontal parameter, d (Fig. 7).

The pendant drop technique requires extreme cleanliness to obtain good results. Here, the needle used for hanging the drop should be cleaned. Also, the needle diameter should not be too small since it reduces the value of $\mathrm{d}$ and can affect the precision of interfacial tension measurements.

\section{Results and discussion}

In this study, three types of plants based natural cationic surfactants, named Olive, Spistan and Prosopis, were used to reduce the IFT of water-oil system and kerosene was used as the oil phase for eliminating the surface active agents present in crude oil.

The profiles of drops just before they are detached from the needle and at the moment of falling, for different aqueous solutions of Olive, Spistan and Prosopis are demonstrated in Fig. 8.

As the surfactant concentration increases, the interfacial tension between the drop and the surrounding oil phase decreases. This is due to the surfactant absorption at the 
Fig. 4 Conductivity of aqueous solution of Olive extract versus surfactant concentration

Fig. 5 Conductivity of aqueous solution of Spistan extract versus surfactant concentration

Fig. 6 Conductivity of aqueous solution of Prosopis extract versus surfactant concentration
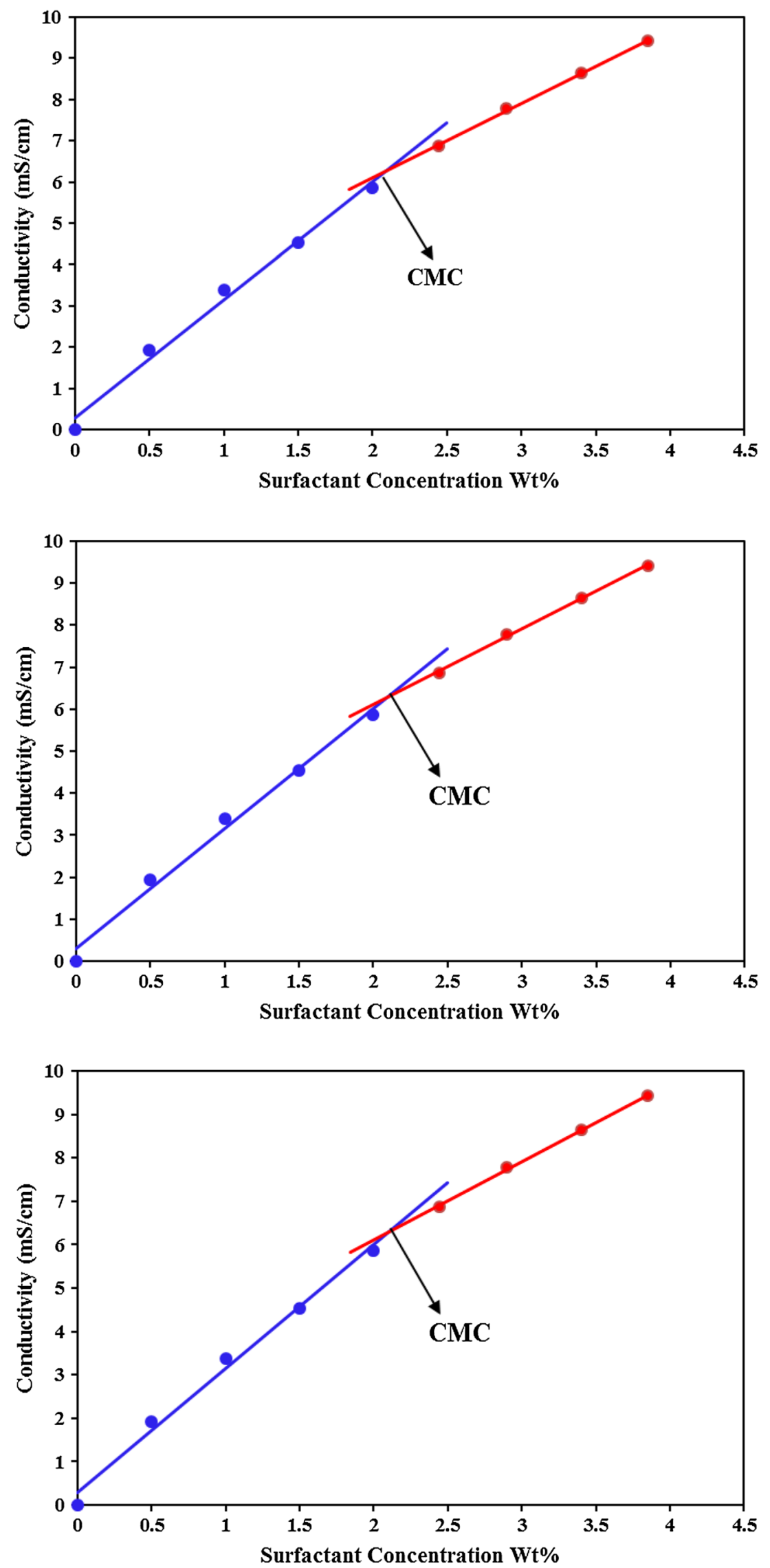
Table 1 Characteristics related to different concentrations aqueous solution of Olive-derived surfactant

\begin{tabular}{lllll}
\hline $\begin{array}{l}\text { Concentration } \\
(\mathrm{wt} \%)\end{array}$ & $\begin{array}{l}\text { Density } \\
\left(\mathrm{g} / \mathrm{cm}^{3}\right)\end{array}$ & $\begin{array}{l}\text { Conductivity @ room } \\
\text { temperature }(\mathrm{mS} / \mathrm{cm})\end{array}$ & PH & IFT $(\mathrm{mN} / \mathrm{m})$ \\
\hline 0 & 0.9943 & 0.0103 & 5.57 & 36.5 \\
0.5 & 0.9962 & 0.62 & 5.38 & 18.93 \\
1 & 0.9983 & 1.2 & 5.32 & 16.3 \\
1.5 & 0.9997 & 1.6 & 5.29 & 15.88 \\
2 & 1.0013 & 1.97 & 5.31 & 14.82 \\
2.44 & 1.0026 & 2.38 & 5.27 & 14.63 \\
2.9 & 1.0042 & 2.74 & 5.28 & 14.39 \\
3.4 & 1.0059 & 3.06 & 5.27 & 14.48 \\
3.85 & 1.0074 & 3.39 & 5.26 & 14.00 \\
\hline
\end{tabular}

Table 2 Characteristics related to different concentrations aqueous solution of Spistan-derived surfactant

\begin{tabular}{lllll}
\hline $\begin{array}{l}\text { Concentration } \\
(\mathrm{wt} \%)\end{array}$ & $\begin{array}{l}\text { Density } \\
\left(\mathrm{g} / \mathrm{cm}^{3}\right)\end{array}$ & $\begin{array}{l}\text { Conductivity @ room } \\
\text { temperature }(\mathrm{mS} / \mathrm{cm})\end{array}$ & PH & IFT $(\mathrm{mN} / \mathrm{m})$ \\
\hline 0 & 0.9943 & 0.0103 & 5.57 & 36.5 \\
0.5 & 0.9964 & 1.93 & 6.96 & 25.07 \\
1 & 0.9986 & 3.38 & 6.91 & 23.9 \\
1.5 & 1.0005 & 4.54 & 6.85 & 22.18 \\
2 & 1.0023 & 5.86 & 6.82 & 21.22 \\
2.44 & 1.0042 & 6.87 & 6.82 & 21.21 \\
2.9 & 1.0057 & 7.79 & 6.81 & 20.93 \\
3.4 & 1.0084 & 8.64 & 6.8 & 20.83 \\
3.85 & 1.0098 & 9.42 & 6.79 & 20.15 \\
\hline
\end{tabular}

Table 3 Characteristics related to different concentrations aqueous solution of Prosopis-derived surfactant

\begin{tabular}{lllll}
\hline $\begin{array}{l}\text { Concentration } \\
(\mathrm{wt} \%)\end{array}$ & $\begin{array}{l}\text { Density } \\
\left(\mathrm{g} / \mathrm{cm}^{3}\right)\end{array}$ & $\begin{array}{l}\text { Conductivity @ room } \\
\text { temperature }(\mathrm{mS} / \mathrm{cm})\end{array}$ & PH & IFT $(\mathrm{mN} / \mathrm{m})$ \\
\hline 0 & 0.9943 & 0.0103 & 5.57 & 36.5 \\
0.5 & 0.9973 & 1.87 & 5 & 23.53 \\
1 & 0.9989 & 3.01 & 4.88 & 18.06 \\
1.5 & 1.0008 & 4.12 & 4.76 & 16.34 \\
2 & 1.0025 & 5.09 & 4.7 & 17.91 \\
2.44 & 1.0037 & 6.07 & 4.63 & 16.12 \\
2.9 & 1.0056 & 6.91 & 4.62 & 15.34 \\
3.4 & 1.007 & 7.63 & 4.61 & 16.25 \\
3.85 & 1.0084 & 8.3 & 4.59 & 15.11 \\
\hline
\end{tabular}

interface between oil and brine. The IFT values between the aqueous solution of addressed natural surfactants and oil were calculated using pendant drop method. In Fig. 9, interfacial tension values for the aqueous solution of Olive oil have been plotted versus surfactant concentration. As

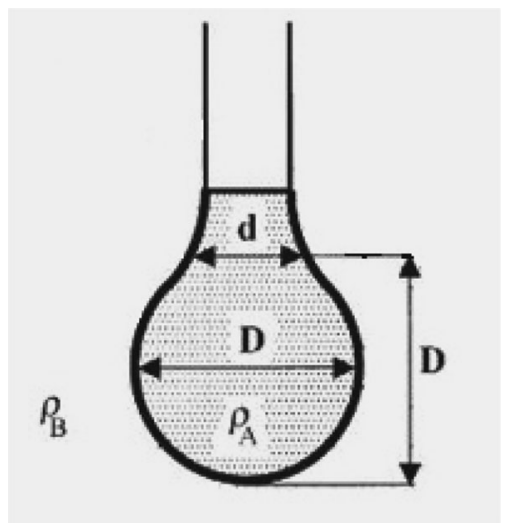

Fig. 7 Geometry of drop illustrating the parameters used in pendant drop method

shown by this graph, $3.85 \mathrm{wt} \%$ of Olive extract have decreased the IFT between kerosene and water from 36.5 to $14 \mathrm{mN} / \mathrm{m}$.

The plots of IFT values for the aqueous solutions of Spistan oil and Prosopis oil against surfactants concentration are shown in Figs. 10 and 11, respectively. As indicated by these graphs, $3.85 \mathrm{wt} \%$ of Spistan extract can reduce the IFT of water-kerosene system from 36.5 to $20.15 \mathrm{mN} / \mathrm{m}$ while Prosopis can decrease that from 36.5 to $15.11 \mathrm{mN} / \mathrm{m}$. It seems that it is difficult to understand why one derivative has better IFT reduction than the other two without knowing the absolute surfactant concentration in each test, but it can be concluded that since the proposed surfactants have been derived under the same conditions for instance time period of drying the leaves of the trees of addressed surfactants, time period of refluxing, refluxing temperature, temperature of the oven, time period of placing the viscous solution, obtained after the refluxing process, in the oven, temperature and humidity of the laboratory and all the other conditions can affect the purity of the obtained natural surfactants, this comparison is true.

As shown in the above figures, little change is observed in the value of interfacial tension beyond the critical micelle concentration point of each natural surfactant since surfactant added in excess of CMC cannot increase the concentration at the water-oil interface and hence participates in micelle formation. Enhanced oil recovery processes would have a better recovery at concentrations above CMC since surfactants can reduce interfacial tension values significantly but it must be noticed that higher concentrations suffer from problems regarding deposition and non-stability of surfactant particles.

Surfactants used in EOR applications should lower IFT to a value with an order of $10^{-3}$, but natural surfactants cannot lower the IFT to this value. As discussed before, 
Fig. 8 Droplet profile at the moment of falling at various surfactant concentrations, a drop shape of aqueous solution of Olive surfactant, b drop shape of aqueous solution of Spistan extract and c drop shape of aqueous solution of Prosopis extract

Olive:

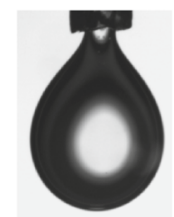

Distilled water

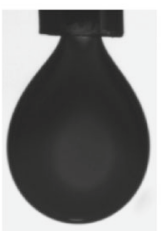

$3.4 \%$

Spistan:

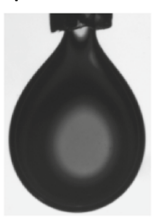

$0.5 \%$

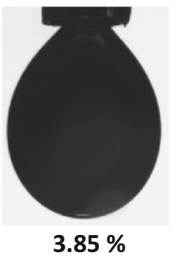

Prosopis:

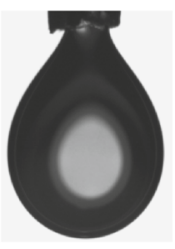

$0.5 \%$
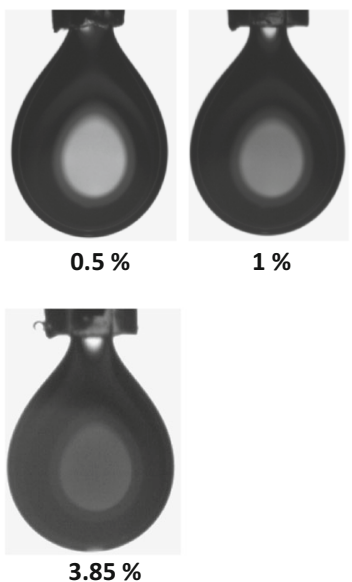

$1 \%$

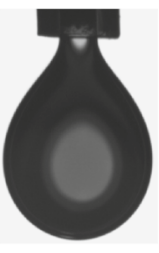

$1.5 \%$

$1 \%$

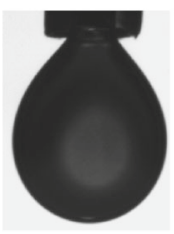

$3.85 \%$

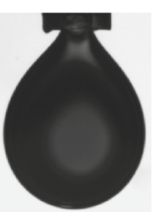

$1 \%$

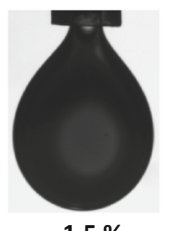

$1.5 \%$
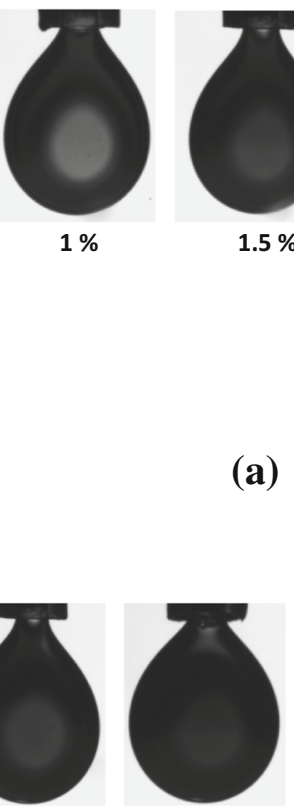

$2 \%$

(b)

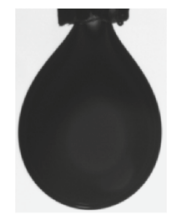

$2.44 \%$

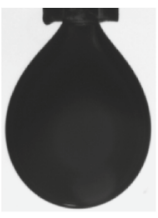

$2.9 \%$

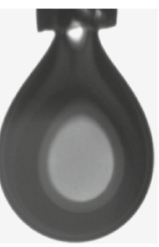

$2 \%$

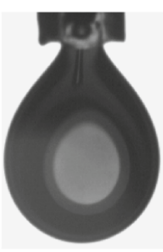

$2.44 \%$

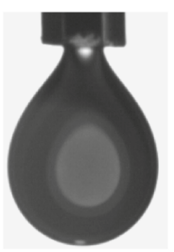

$2.9 \%$

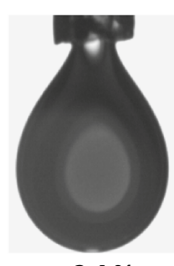

$3.4 \%$

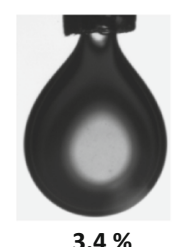

$.4 \%$

\section{(c)}

because of environmental fates and also the price of industrial surfactants, natural-based surfactants can be used instead of industrial surfactants and many researchers have studied these kinds of surfactants in recent years. In addition, additives can be used with natural surfactants so that these surfactants could lower the IFT to an appropriate value. These additives have lower price than the industrial surfactants.

\section{Conclusions}

In this study three new natural-based cationic surfactants produced from the leaves of Olive, Spistan and Prosopis were studied for their ability in reducing the interfacial tension of water and oil system. Based on the results obtained from this research, following conclusions can be drawn: 
Fig. 9 Plot of interfacial tension versus Olive surfactant concentration at room temperature

Fig. 10 Plot of interfacial tension versus Spistan surfactant concentration at room temperature

Fig. 11 Plot of interfacial tension versus Prosopis surfactant concentration at room temperature
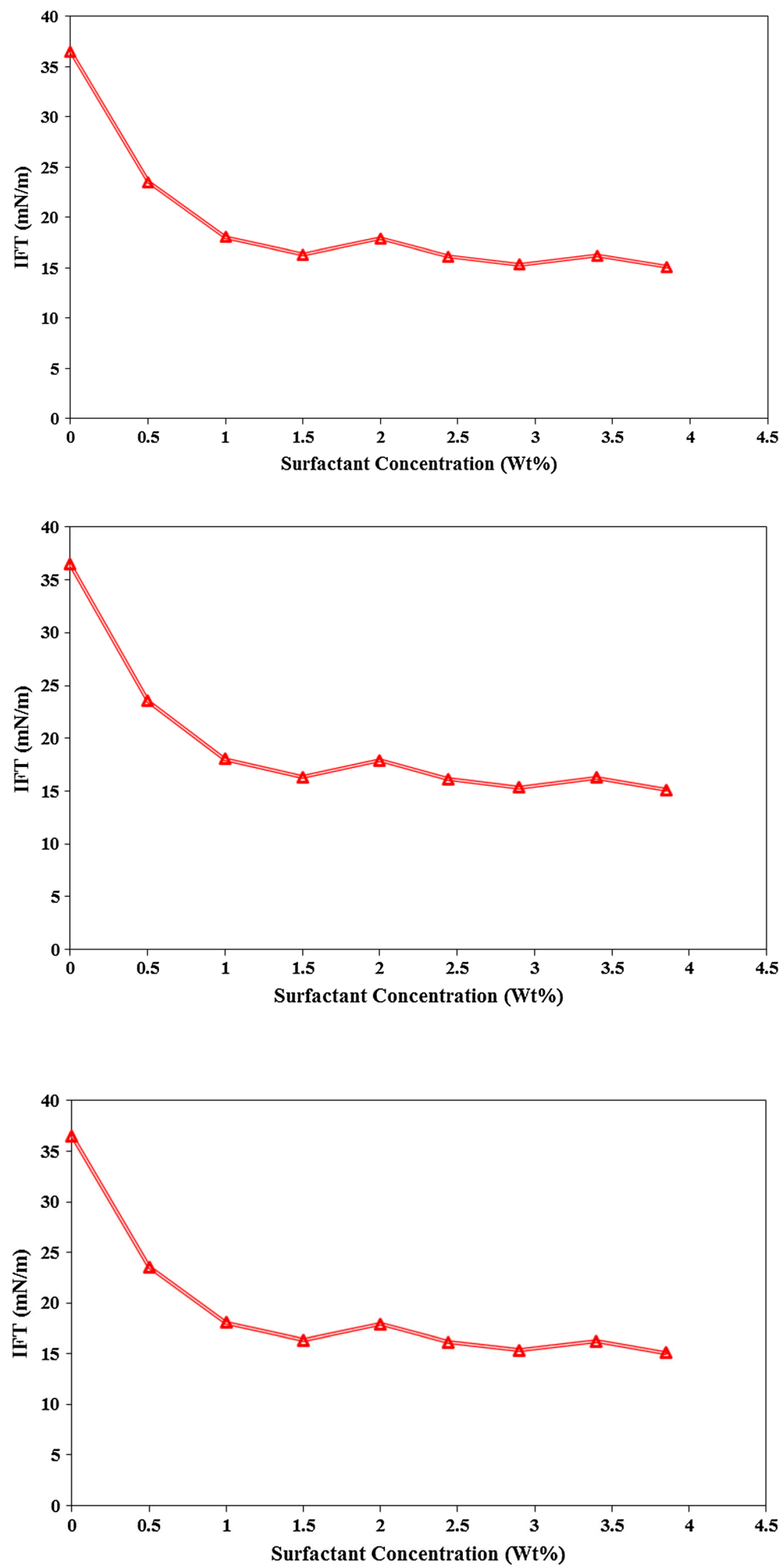
1. The results demonstrated that a surfactant containing $3.85 \mathrm{wt} \%$ of Olive extract could reduce the IFT between kerosene and distilled water from 36.5 to $14 \mathrm{mN} / \mathrm{m}$, while the same amount of Spistan and Prosopis extracts lowered the IFT from 36.5 to $20.15 \mathrm{mN} / \mathrm{m}$ and 36.5 to $15.11 \mathrm{mN} / \mathrm{m}$, respectively.

2. Olive-derived surfactant has a critical micelle concentration of about $1.95 \%$ by weight, while Spistanderived surfactant and Prosopis-derived surfactant have CMCs of about 2.1 and $2.3 \%$, respectively. The CMC values of these natural surfactants can be used in future oil recovery studies.

3. PH of deionized water was considered as the reference. Solution with a PH value more than referenced value is in basic range and solutions with a $\mathrm{PH}$ value lower than referenced value is in acidic range so, the Spistan surfactant solutions are in basic range, while the Olive and Prosopis surfactants solutions are in acidic range.

4. Olive, Spistan and Prosopis plants are now widely available in several regions, so that high surface activity surfactants can be obtained. According to this in addition to the low price of generating plant-derived surfactants, the feasibility of using these kinds of surfactants in future oil recovery processes is of major concern.

Open Access This article is distributed under the terms of the Creative Commons Attribution License which permits any use, distribution, and reproduction in any medium, provided the original author(s) and the source are credited.

\section{References}

Aberoumand A (2011) Preliminary Evaluation of Some Phytochemical and Nutrients Constituents of Iranian Cordia myxa Fruits. Int J Agric Food Sci 1(2):30-33

Ahmadi MA, Shadizadeh SR (2012) Adsorption of novel nonionic surfactant and particles mixture in carbonates: enhanced oil recovery implication. Energy Fuels 26:4655-4663

Ahmadi MA, Shadizadeh SR (2013) Experimental investigation of adsorption of a new nonionic surfactant on carbonate minerals. J Fuel 104:462-467

Ahmadi MA, Arabsahebi Y, Shadizadeh SR, Shokrollahzadeh Behbahani S (2014) Preliminary evaluation of mulberry leafderived surfactant on interfacial tension in an oil-aqueous system: EOR application. Fuel 117:749-755

Alibes X, Berge Ph (1983) Valorizacion de los subproductos Del olivar como alimentos para los rumiantes. FAO, Rome

Deymeh H, Shadizadeh SR, Motafakkerfard R (2012) Experimental investigation of Seidlitzia rosmarinus effect on oil-water interfacial tension: usable for chemical enhanced oil recovery. Sci Iran 19(6): 1661-1664

Herd MD (1992) Interfacial tensions of microbial surfactants determined by real-time video imaging of pendant drops. In: SPE 24206 presented at SPE improved oil recovery symposium

Pordel Shahri P, Shadizadeh SR, Jamialahmadi M (2012) Applicability test of new surfactant produced from Zizyphus Spina Christi leaves for enhanced oil recovery in carbonate reservoirs. J Jpn Pet Inst 55(1):27-32

Rigano L, Lionetti N (2009) Quillaja triterpenic saponins can act as a natural emulsifier and dispersing agent to offer a real alternative to synthetic surfactants. J SPC 82(11):1-4

Spildo K, Johannessen AM, Skauge A (2012) Low salinity waterflood at reduced capillarity. In: Eighteenth SPE improved oil recovery symposium held in Tulsa. Oklahoma, USA, pp 14-18 